\title{
The burden, pattern and factors that contribute to periportal fibrosis in HIV-infected patients in an S. mansoni endemic rural Uganda.
}

\author{
Ponsiano Ocama ${ }^{1}$, Kenneth Christopher Opio ${ }^{1}$, Emmanuel Seremba $^{1}$, Paul Ajal ${ }^{2}$, \\ Betty Stephanie Apica ${ }^{1}$, Emmanuel Odongo Aginya $^{3}$
}

1. Department of Medicine, Makerere University College of Health Sciences, Kampala, Uganda

2. Pakwach Health Centre IV, Pakwach, Nebbi District, Uganda

3. Gulu University Medical School.

\begin{abstract}
Introduction: Both Human Immunodeficiency Virus (HIV) and S.mansoni infections are common in Uganda and can cause liver disease. No study has determined co-infection significance in Uganda. We carried out a study on the burden, pattern and factors that contribute to peri-portal fibrosis (PPF) in HIV infected patients attending a Primary healthcare setting at Pakwach. Methodology: We conducted a cross-sectional study in the HIV clinic at Pakwach health centre IV. Data on demographics, contact with the Nile, $\mathrm{CD}^{+}$cell count, ART and alcohol use were collected. Urinary Circulating Cathodic Antigen (CCA), was done for S. Mansoni detection. Liver scan was done for presence and pattern of PPF. HBsAg testing was performed on all participants. Data was analyzed using Stata Version 10.

Results: We enrolled 299 patients, median age 39 years (IQR 16), most were female, 210 (73\%). Overall, 206 (68.9\%) had PPF, majority $191(92.7 \%)$ had pattern c, either alone (63 participants) or in combination with pattern d (128 participants). Age of 30-50 years was significantly associated with PPF (OR 2.28 p-value-0.003)

Conclusion: We found high prevalence of $S$. mansoni and PPF in the HIV infected population and age was a significant factor for PPF. We recommend all HIV infected patients be examined routinely for $S$. mansoni infection for early anti-schistosomal treatment. Keywords: HIV, S.Mansoni, peri-portal fibrosis.

DOI: https://dx.doi.org/10.4314/ahs.v17i2.2

Cite as: Ocama P, Opio KC, Seremba E, Ajal P, Apica BS, Aginya EO. The burden, pattern and factors that contribute to periportal fibrosis in HIV-infected patients in an S. mansoni endemic rural Uganda. Afri Health Sci. 2017;17(2): 301-307. bttps:// dx.doi.org/10.4314/abs.v17i2.2
\end{abstract}

\section{Introduction}

Schistosoma mansoni is highly endemic in Uganda especially along the shores of river Nile and Lake Albert. Pakwach is found at a point several kilometers from where the river exits Lake Albert proceeding Northwards to South Sudan. The people of Pakwach rely on the Nile for fishing as the main source of income. They also use the water for drinking, bathing and washing clothes. This in a sense makes them very vulnerable to $S$. mansoni infection. A previous study showed a prevalence of more than $70 \%$ in
Corresponding author:
Ponsiano Ocama,
Department of Medicine
Makerere University,
College of Health Sciences
P.O. Box 7072
Kampala, Uganda
Phone: +256772421190
Email: ponsiano.ocama@gmail.com

the population around the lake Albert area, and Pakwach is within this region. ${ }^{1}$ The prevalence is even higher in the younger age group where over $90 \%$ of the children aged 10-14 years are infected. ${ }^{2}$ On the other hand, the prevalence of HIV in Pakwach is 11\%, higher than the $4.7 \%$ reported for Nebbi District where Pakwach is located. (Nebbi District 5 year Strategic plan, 2012). Because of the high endemicity of $S$. mansoni and the fact that this infection is acquired earlier in life, we expect that among HIV infected persons (where most infections are sexually transmitted in adulthood) the proportion with co-infection is high.

Schistosoma mansoni causes liver disease by producing periportal fibrosis. Similarly, HIV produces liver diseases by several mechanisms including hepatotoxicity caused by various drugs used in treatment of HIV and/or opportunistic infections, viral hepatitis $\mathrm{B}, \mathrm{C}$ and herpes virus, opportunistic infections such as cryptosporidiosis and tuberculosis and malignancies such as lymphomas and $\mathrm{Ka}$ - 
posi's sarcoma. ${ }^{3}$ Although the effects of HIV and viral hepatitis B, C and other infections have been studied extensively, co-infection with the neglected tropical disease, Schistosomiasis is not well studied especially in areas where both infections are highly endemic as in Pakwach, Nebbi District.

We determined the prevalence of $S$. mansoni among HIV-infected patients as well as the presence and extent of periportal fibrosis and factors associated with periportal fibrosis in this patient population.

\section{Methodology}

We conducted a cross-sectional study with analysis of factors associated with periportal fibrosis. The study was conducted in Pakwach health centre IV in the HIVclinic. This is a rural health centre located at the shore of river Nile serving 5 sub-counties of Pakwach, Pakwach town council, , Panyimur, Panyango and Wadelai. Panyimur and Wadelai sub-counties are the furthest from the health center which is located in Pakwach town. The populations within these sub-counties rely on the Nile for their livelihood.

Pakwach health centre has a bed capacity of 62 , admits about 5,000 patients a year and runs an out-patient clinic daily, from Monday to Friday, receiving 80 patients every day. Approximately 120 patients present with episodes of gastrointestinal bleeding annually that is attributed to schistosomiasis.

The HIV clinic runs two times a week and on average 80 patients attend the HIV clinic on each of the clinic days. The overall clinic population is approximately 4,000 patients and about half of these are on antiretroviral therapy (ART).

The centre has a laboratory with a trained laboratory technician and a radiographer. The radiographer was trained to conduct sonographic analysis using an established protocol on severity of liver disease in Schistosomiasis using the modified Niamey protocol. ${ }^{4}$

\section{Procedure}

All adult patients (18 years and above) were eligible to participate in the study. Because of the numbers, the first 20 patients registered on each clinic day who fulfilled the criteria for inclusion and accepted to participate in the study were enrolled after signing an informed consent form.
A questionnaire was administered to collect demographic data and other information including age, gender, area of residence (sub-county), occupation, history of contact with water bodies, previous history of yellow eyes. Other information collected included $\mathrm{CD} 4^{+}$cell count (at ART initiation and current if applicable), ART use and duration if applicable, opportunistic infections ever suffered and any other medications. History of alcohol use, treatment for $S$. mansoni infection using Praziquantel either when found infected or as part of mass drug administration (MDA) were also ascertained.

Blood draws were made to examine for LFTs and CD4 ${ }^{+}$ cell count if the last test was more than 6 months. To demonstrate $S$. mansoni infection, all patients were screened using the Circulating-Cathodic-Antigen (CCA) urine dipstick test (Rapid Medical Diagnostics, Pretoria, RSA) . This test had been evaluated in Uganda and Kenya and was found to have high sensitivity and specificity especially in field studies. ${ }^{5,6}$ All participants were then subjected to ultrasound scan of the liver to determine peri-portal fibrosis using the modified Niamey protocol to determine peri-portal fibrosis and its severity. ${ }^{4} \mathrm{HBsAg}$ testing was performed on all participants using a rapid one-step test for HBsAg (Eurostrip, Euromedic Equipment, Netherlands) following the manufacturer's instructions.

Data was entered into a computer using epidata 10 and exported to stata 10 version for analysis. Proportions were used to determine prevalence and grading of periportal fibrosis was done using the WHO staging and the modified Niamey protocol to determine peri-portal fibrosis and its severity, where grades a,b represents no or minimal fibrosis, severity progressing through $\mathrm{c}, \mathrm{d}$ and $\mathrm{f}$ (most severe). In addition, $\mathrm{x}, \mathrm{y}, \mathrm{z}$ was reported when periportal fibrosis was in addition to other pathologies- cirrhosis, fatty liver and other hepato-biliary pathologies. ${ }^{4,7}$ Frequencies and proportions were determined describing the socio-demographic characteristics of the study population, the different patterns of peri-portal fibrosis, and the main study outcome which was presence or absence of peri-portal fibrosis. ${ }^{4}$

Possible factors for PPF were analyzed in bivariate model and those with $\mathrm{p}$-value $<0.2$ were analyzed in a multivariate model to determine significant factors of PPF. This was done by developing an interaction term. Odds ratios 
were used to express the extent to which these factors relate to the presence or absence of peri-portal fibrosis and p-values $<0.05$ were considered significant.

The study was carried out following international and national guidelines for research on human subjects and in accordance with the Helsinki Declaration. It was approved by School of Medicine Research and Ethics committee (SOMREC) of Makerere University College of Health Sciences and the National Council of Sciences and technology (UNCST). Test results were passed onto the attending clinicians for management of the patients.

\section{Results}

\section{Socio-demographic characteristics}

During the months of June and July 2014, we enrolled 299 patients who fulfilled the criteria for inclusion and who had complete data, median age was 39 years (IQR 16). The youngest participant was 18 years while the oldest was 69 years of age.

There was a female predominance 210 (73\%). Because of the proximity of Pakwach town council and Pakwach sub-county, the 2 sub-counties were considered together and majority $(75 \%)$ of the participants came from this area and almost all had been in contact with the Nile; (Table 1).

Table 1. Socio-demographic characteristics of 299 HIV infected in rural Pakwach Health Centre IV

\begin{tabular}{|l|l|l|}
\hline Variable & Frequency (n) & Proportion (\%) \\
\hline Age range & & \\
$18-29$ & 51 & 17.1 \\
$30-50$ & 180 & 60.2 \\
$>50$ & 68 & 22.7 \\
Male & 81 & 27.1 \\
Female & 218 & 72.9 \\
Tribe & & \\
Alur & 295 & 98.7 \\
Others & 4 & 1.3 \\
Subcounty & 227 & 75.9 \\
Pakwach & 67 & 22.4 \\
Panyango & 3 & 1 \\
Panyimur & 2 & 0.6 \\
Wadelai & \\
Contact with the Nile & & \\
Yes & 288 & 96.3 \\
No & 11 & 3.7 \\
Alcohol use & 144 & 48.2 \\
Yes & 155 & 51.8 \\
No & \\
\hline
\end{tabular}

\section{Peri-portal fibrosis}

Of 299 participants with complete data, 206 (68.9\%) of the participants had peri-portal fibrosis of various grades.
Most of the participants with PPF, 191 (92.7\%) had pattern c PPF, either alone (63 participants) or in combination with pattern d (128 participants); Table 2 . 
Table 2. Burden and Ultrasound patterns of peri-portal fibrosis (PPF) in 299 HIV infected patients, using the Modified Niamey protocol

\begin{tabular}{|l|l|l|}
\hline Variable & N & \% \\
\hline $\begin{array}{l}\text { Periportal fibrosis } \\
\text { Present (c-f) }\end{array}$ & 206 & 68.9 \\
Patterns of PPF & & \\
a,b (No/Minimal PPF) & 93 & 31.14 \\
$\mathrm{c}$ & 63 & 21.1 \\
$\mathrm{c}+\mathrm{d}$ & 128 & 42.8 \\
$\mathrm{~d}$ & 2 & 0.7 \\
$\mathrm{~d}+\mathrm{e}$ & 9 & 3 \\
$\mathrm{~d}+\mathrm{x}$ & 2 & 0.7 \\
$\mathrm{f}$ & 2 & 0.7 \\
\hline
\end{tabular}

Factors associated with peri-portal fibrosis significantly associated with peri-portal fibrosis. (Table 3) In bivariate analysis, age and sub-county of origin were This pattern was repeated in multivariate model.

Table 3. Factors associated with peri-portal fibrosis (PPF) at ultrasound scan in 299 HIV infected patients at Pakwach Health Centre in rural Uganda

\begin{tabular}{|c|c|c|c|c|}
\hline Parameters & $\begin{array}{l}\text { No PPF: N=93 } \\
\text { n }(\%)\end{array}$ & PPF: $N=206$ n (\%) & Unadjusted OR & P-value \\
\hline $\begin{array}{l}\text { Age 30-50 years } \\
\text { Yes } \\
\text { No } \\
\text { Gender } \\
\text { Male } \\
\text { Female } \\
\text { Sub-county of origin } \\
\text { Pakwach } \\
\text { Panyango } \\
\text { Panyimur } \\
\text { Wadelai } \\
\text { Infection with } \\
\text { S. mansoni * } \\
\text { Yes } \\
\text { No } \\
\text { CD4 counts** } \\
0-200 \\
201-500 \\
\text { >500 } \\
\text { HBsAg positive } \\
\text { Yes } \\
\text { No } \\
\text { TDF and/or 3TC } \\
\text { ART combination } \\
\text { Yes } \\
\text { No } \\
\text { Alcohol use } \\
\text { Yes } \\
\text { No }\end{array}$ & $\begin{array}{l}45(48) \\
48(52) \\
\\
21(22.6) \\
76(81.7) \\
74(79.6) \\
15(16.1) \\
0 \\
0 \\
85(32) \\
8(23)\end{array}$ & $\begin{array}{l}137(66) \\
69(34) \\
60(29.1) \\
142(68.9) \\
157(76.2) \\
52(25.2) \\
3(100) \\
2(100) \\
\\
183(68) \\
23(74) \\
\\
22(60) \\
66(74) \\
43(69) \\
18(67) \\
183(69) \\
174(84) \\
32(16) \\
97(70) \\
103(68)\end{array}$ & $\begin{array}{l}1.19(0.77-1.84) \\
0.89(0.38-2.07) \\
0.96(0.49-1.90) \\
1.03(0.63-1.69)\end{array}$ & 0.43 \\
\hline
\end{tabular}




\section{Discussion}

Our study found PPF fibrosis in $68.9 \%$ of HIV infected patients in this primary care clinic. Peri-portal fibrosis was associated with age of more than 30 years and sub-county of origin. Ultrasound patterns $c, c+d, d$, that indicate mild to moderate PPF were the most common patterns observed.

Pakwach and other areas around the lake Albert region have reported very high prevalence of $S$. mansoni. The study also showed that the prevalence of $S$. mansoni estimated from participants who were CCA positive plus those who had received treatment for $S$. mansoni using praziquantel was $89.6 \%$. This finding has been corroborated before in several studies that clearly defined the Albertine area with high prevalence of S. mansoni. Eradication of $S$. mansoni had been thwarted in this region by the fact that the livelihood of the population is derived from the river Nile. The fishing practice in this region is such that men go fishing at night with fishing boats that are open roof and without any toilet facilities. Whenever there is need therefore, fecal matter is deposited in the Nile. Because of the high prevalence of the infection, this propagates the transmission of this infection to the population. The river has also been used as source of water for drinking, bathing and washing clothes without any purification process. Even among those who have not had direct contact with the river, exposure can still occur at home from use of water sourced from the river Nile.

The high prevalence of peri-portal fibrosis seen in this study indicates a high level of liver associated morbidity. We found most participants had either mild or moderate peri-portal fibrosis, which is supported by other findings as being associated with a low prevalence of upper gastrointestinal bleeding.

However because we did not carry out any endoscopy, we could not assess the presence of varices which would determine the risk of future variceal bleeding. A separate study has been designed to look at endoscopic findings.

Nevertheless, a personal communication from Pakwach health centre director indicates that between 2-3 cases of upper gastrointestinal tract (UGIT) bleeding present to this centre every week and with very high mortality.

We found that $68 \%$ of the participants with peri-portal fibrosis had evidence of $S$. mansoni. This means there could have been other etiological factors explaining periportal fibrosis. HIV infection and other infections such as tuberculosis have been shown to cause peri-portal fibrosis as well. ${ }^{8}$

Age of over 30 years and sub-county of origin were the main factors associated with risk of PPF. Peri-portal fibrosis is generally a slow process and could therefore occur later from time of exposure. Although fishing in this community begins from as early as 10 years and infection occurs early in life, the process of scarring takes time to occur and is seen at a later time in life. The sub-county of origin being significantly associated with PPF is probably because most participants were from these sub-counties.

Participants from this HIV clinic were mainly young adults of female gender. Considering that the median time to progression of HIV infection has been reported as 10 years, we can estimate that the majority of our participants got infected more than 10 years prior. ${ }^{9}$ This indicates that in this community HIV infection is common among adults below the age of 30 years. Generally, most HIV clinics report females more than males and this is more likely due to health care seeking behavior, which explains our findings..$^{10}$ However, gender disproportion in our study could have biased our results since males have been reported to have more severe peri-portal fibrosis than females. ${ }^{11}$

These study findings have wider implications. Schistosoma mansoni is common in many parts of Uganda, especially in areas along river Nile, Lake Kyoga and Lake Albert. The incidence of HIV has been shown to be higher among the fisher folks around lake Victoria, mainly associated with drinking of alcohol. This behavior is common in all the fisher folks in the country and the results of this study have implications to all of them as well..$^{13}$

\section{Limitations}

First, the study was only conducted among HIV infected patients and this could have affected the finding of peri-portal fibrosis as some studies have shown that HIV tends to reduce the egg production of schistosomiasis. ${ }^{12}$ Since the reactions leading to peri-portal fibrosis in schistosomiasis are mainly a result of reactions to the eggs, there could be an underestimate of peri-portal fibrosis. A study carried out in Tanzania showed PPF grades were similar in both patients who had HIV and Schistosomiasis co-in- 
fection compared to those with schistosomiasis mono-infection. ${ }^{14}$

The second limitation is that the CD4 Cell count was not available for all the participants as only 188 participants had their CD4 cell count recorded. This made it difficult to draw significant conclusion on the effect of CD4 count on peri-portal fibrosis and could have led to an insignificant level seen in the study.

\section{Conclusion}

Our study found high prevalence of $S$. mansoni and periportal fibrosis in the HIV infected population in the rural health centre IV in the Albertine region of Uganda and age is a significant factor in the development of severe periportal fibrosis. We recommend that all HIV infected patients in this area be checked routinely for $S$. mansoni infection either using the CCA method or even stool examinations to determine presence or absence of schistosomiasis. Treatment given earlier on to prevent this detrimental consequence of infection would then be important. The alternative is to continue giving routine praziquantel administration at regular intervals to prevent the effect of peri-portal fibrosis.

\section{Acknowledgment}

The project was supported by the MESAU-MEPI Programmatic Award through Award Number 1R24TW008886 from the Fogarty International Center. The Content is solely the responsibility of the authors and does not necessarily represent the official views of the Fogarty International Center or the National Institutes of Health

\section{Competing interests}

The authors declare no competing interests.

\section{Contributions of the authors}

All authors reviewed the manuscript and gave significant contributions to the paper.

PO developed the proposal, supervised the research work and data analysis, participated in initial manuscript draft and approved the final manuscript. $\mathrm{KCO}$, developed the proposal, analyzed the data and wrote the initial drafts of the manuscript, ES, PA, BSA made extensive review of the manuscript and approved the final manuscript, EOA participated in developing the proposal and approved the manuscript.

\section{References}

1. Kabatereine NB, Kemijumbi J, Ouma JH, et al. Epidemiology and morbidity of Schistosoma mansoni infection in a fishing community along Lake Albert in Uganda. Trans R Soc Trop Med Hyg. 2004;98:711-8.

2. Kabatereine NB, Ariho C, Christensen NO. Schistosoma mansoni in Pachwach, Nebbi District, Uganda, 40 years after Nelson. Trop Med Parasitol 1992; 43:162-6 PubMed . 3. Soriano V, Barreiro P, Sherman KE. The changing epidemiology of liver Disease in HIV patients. AIDS Rev 2013;15(1):25-31. PubMed

4. El Scheich T, Holtfreter MC, Ekamp H, et al. The WHO ultrasonography protocol for assessing hepatic morbidity due to Schistosoma mansoni. Acceptance and evolution over 12 years Parasitol research. 2014;113:3915-25.

5. Stothard JR, Kabatereine NB, Tukahebwa EM, et al. . Use of circulating cathodic antigen (CCA) dipsticks for detection of intestinal and urinary schistosomiasis. Acta Trop. 2006;97:219-28 PubMed .

6. Shane HL, Verani JR, Abudho B, et al. Evaluation of Urine CCA Assays for Detection of Schistosoma mansoni Infection in Western Kenya. PLOS Negl Trop Dis 2011;5:e951.

7. Berhe N, Geitung JT, Medhin G, Gundersen SG. Large scale evaluation of WHO's ultrasonographic staging system of schistosomal peri-portal fibrosis in Ethiopia. Trop Med Int Health. 2006;11:1286-94 PubMed .

8. Abdel-Kader S, Amin M, Hamdy H, Mohran Z, Abbas MM. Causes of minimal peri-portal fibrosis in Egypt. $J$ Egypt Soc Parasitol 1997 Dec;27(3):919-24.

9. Morgan D, Mahe C, Mayanja B, Okongo JM, Lubega $\mathrm{R}$, Whitworth JA. HIV-1 infection in rural Africa: is there a difference in median time to AIDS and survival compared with that in industrialized countries? AIDS 2002; 16:597-603 PubMed .

10. Mills EJ, Ford N, and Mugyenyi P. Expanding HIV care in Africa: making men matter. The Lancet 2009; 374:275-6 PubMed .

11. Mohamed-Ali Q, Elwali NE, Abdelhameed AA, et al. Susceptibility to peri-portal (Symmers) fibrosis in human Schistosoma mansoni infections: evidence that intensity and duration of infection, gender, and inherited factors are critical in disease progression. J Infec Dis. 1999;180:1298306 PubMed .

12. Secor WE, Karanja DMS, Cooley DG. Interactions between schistosomiasis and human immunodeficiency vi- 
rus in Western Kenya. Mem Inst Oswaldo Cruz 2004;99:93- Uganda. PLoS ONE, 2014; 9: e9493214. Mazigo HD, 5. Dunne DW, Morona D, et al. Peri-potral fibrosis, liver

13. Kiwanuka N, Ssetaala A, Nalutaaya A, et al. (2014) High Incidence of HIV-1 Infection in a General Popand spleen sizes among $S$. mansoni mono or co-infected individuals with human immunodeficiency virus-1 in ulation of Fishing Communities around Lake Victoria,

fishing villages along Lake vistoria shores, Northwestern, Tanzania. Parasit Vectors 2015;8:260. 